\title{
Research on a bifurcation location algorithm of a drainage tube based on 3D medical images
}

\author{
Qiuling Pan ${ }^{1}$, Wei Zhu', Xiaolin Zhang ${ }^{1}$, Jincai Chang ${ }^{1 *}$ (D) and Jianzhong Cui ${ }^{2^{*}}$
}

\begin{abstract}
Based on patient computerized tomography data, we segmented a region containing an intracranial hematoma using the threshold method and reconstructed the 3D hematoma model. To improve the efficiency and accuracy of identifying puncture points, a point-cloud search arithmetic method for modified adaptive weighted particle swarm optimization is proposed and used for optimal external axis extraction. According to the characteristics of the multitube drainage tube and the clinical needs of puncture for intracranial hematoma removal, the proposed algorithm can provide an optimal route for a drainage tube for the hematoma, the precise position of the puncture point, and preoperative planning information, which have considerable instructional significance for clinicians.
\end{abstract}

Keywords: Multitube drainage tube, Bifurcation localization algorithm, 3D medical image, Path planning, Intracranial hematoma

\section{Introduction}

Trauma can cause blood vessels to burst in the brain or between the skull and brain tissue. Subsequently, an intracranial hematoma may form from blood pooling up in the brain or between the skull and brain, which compresses the brain tissue. An intracranial hematoma is a common but serious secondary damage mode of craniocerebral injury. The incidence of closed craniocerebral injury is approximately $10 \%$, while the incidence of severe craniocerebral injury is approximately $40 \%-50 \%$. The complications of intracranial hematomas include their effect on cerebral blood flow, cerebral hernia, cerebral edema, and Cushing's reaction, which can seriously damage brain tissue, and some of these damage modes are irreversible and life-threatening [1-4]. Puncture and ablation of the intracranial hematoma is a widely used medical method. With the increasingly busy modern life, heavy work stress, mental pressure, and lack of physical labor, the

\footnotetext{
* Correspondence: jincai@ncst.edu.cn; jzhcui0001@163.com

${ }^{1}$ College of Sciences, North China University of Science and Technology, Tangshan 063210, Hebei, China

2Department of Neurosurgery, Tangshan Gongren Hospital, Tangshan 063000, Hebei, China
}

incidence rates show that the age of onset of this condition is decreasing. Once it occurs, the disease places a burden on individuals and families, so all countries and the international community have attached considerable importance to it [2]. The advantages of puncture operation include effective application, high security, quick recovery, short operating time, and relatively few postoperative complications [5-7]. At present, the methods of positioning such as the stereotactic technique [8] and neuronavigation technique [9] mainly involve a freehand technique for insertion of a drainage tube, which is based on fixed anatomical landmarks, does not consider individual variations, and often exhibits insufficient precision. Hence, a time-efficient and low-cost technique to localize the hematoma puncture point and to provide path planning will be beneficial, especially when highly sophisticated and expensive navigation systems cannot be made available in developing regions. In the present study, according to a dataset of brain computerized tomography $(\mathrm{CT})$ images, we reconstructed the $3 \mathrm{D}$ model of a patient's brain by using a 3D Slicer software [10-14] and extracted the area of intracranial hematoma as well. To quickly find the best 
puncture point in theory, an algorithm based on kmeans clustering [15] was proposed to optimize the search space, eliminate redundant computation, effectively sort out the search space, and reduce the possibility of particles falling into a local extremum [16]. Then, the algorithm is improved from the viewpoint of the search mode, and the point cloud search algorithm of adaptive weighted particle swarm optimization [17-19] is proposed, which greatly reduces the calculation time and the number of iterations of the algorithm. In the experimental environment, the algorithm proposed in this study can greatly improve the search efficiency of this method in obtaining the global optimal solution. Compared with the direct optimization algorithm [20], the average maximum acceleration ratio is $1288.67 \%$. According to the characteristics of the multitube drainage tube [21] and the clinical needs of the puncture process for intracranial hematoma removal, the bifurcation location algorithm provides the precise position of the puncture point, the optimum location of probes, and the best route under ideal conditions. In this study, the 3D model is effectively combined with the multitube drainage tube; furthermore, a preoperative simulation is proposed, which can provide significant guidance and value in formulating the puncture operation plan and decreasing the risk of blindness.

\section{Segmentation of the intracranial hematoma}

In clinical medicine, for patients with an intracranial hematoma, most hospitals conduct CT, hematoma location, puncture marking, followed by other processes $[5,6]$. We used the 3D Slicer software to implement three-dimensional reconstruction based on brain CT data. This approach can greatly reduce the treatment time and improve efficiency. 3D Slicer software can reconstruct anatomical parts such as the hematoma, blood vessels, skull, and nerve bundles. In this way, doctors can avoid important parts such as blood vessels and nerve bundles when they mark the puncture points and accurately and efficiently puncture the hematoma, thus reducing treatment time [12-14]. The 3D Slicer program is a software platform for the analysis (including registration and interactive segmentation) and visualization (including volume rendering) of medical images and for research in image guided therapy. The platform originated in an MSc thesis program in 1998 and is jointly sponsored by the Surgery Plan Laboratory at Brigham and Women's Hospital and the Artificial Intelligence Laboratory at the Massachusetts Institute of Technology [10-12].

\section{Hematoma modeling}

After the CT data have been preprocessed, threedimensional reconstruction of the brain can be realized to obtain a three-dimensional image. In 3D Slicer, the region of interest (ROI) function is used to extract the hematoma area in the image, and the Segmentations module is selected to model the hematoma, providing models in the STL and OBJ formats to extract the "optimal external central axis" needed in this study.

\section{Hematoma location locking}

First, the Paint function in Slicer is applied to draw the hematoma, and then, the Fast Marching Method is used to draw the models of the intracerebral hematoma within a three-dimensional sphere, which is of great help in the follow-up work. Finally, the three-dimensional sphere is initialized by the Fast Marching function. Because the mask area around the hematoma is very large after initialization, the mask is narrowed by adjusting the slider for Segment volume, and the position of the hematoma is locked (Fig. 1).

\section{Applying the threshold method to segment the hematoma}

We select the Threshold function, in which the threshold is set to 50-100, set the selected intensity range to an editable intensity for the mask, and then, convert the masked area into a 3D model. For multiple small masks that appear in CT images, we use the Smoothing function to make the hematoma easier to segment by removing protrusions and filling holes to make the segmentation boundaries smoother. After smoothing, we use the Islands function to segment the hematoma from the numerous masks. We select the Keep selected island and click on the hematoma to segment it (Fig. 2). For specific modeling steps and methods, please refer to ref. [12].

\section{Methods \\ Basic concept and definition}

As an effective tool to describe the morphological characteristics of the geometry, the center axis has many advantages; for example, it can describe the geometry information inside the model and at any position of the boundary [22-24]. Furthermore, it provides a method to intuitively describe the geometric characteristics of a complex model and represent the topological relation of the model. The definition of the external central axis is as follows: Let $\Omega$ be a model in $n$-dimensional Euclidean space $R^{n}$, and assume it is homeomorphic to a $3 \mathrm{D}$ closed sphere; we define $\Omega$ as an $n-D$ model. The external center axis of $\Omega$ is the set of the centers of the maximum circumscribed spheres [25]. Inspired by the 


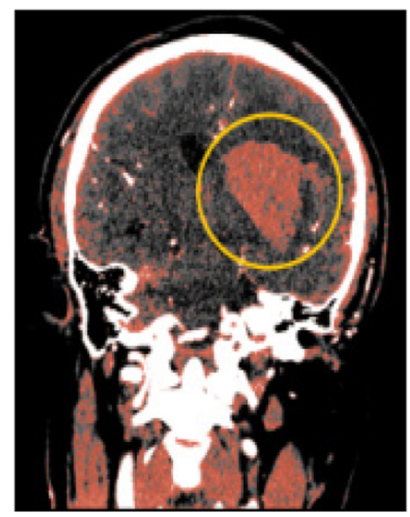

a

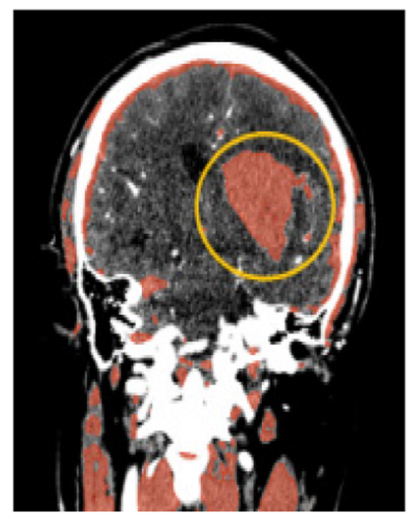

b

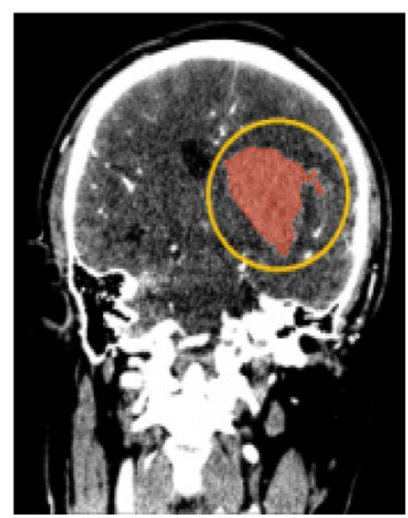

$\mathrm{C}$

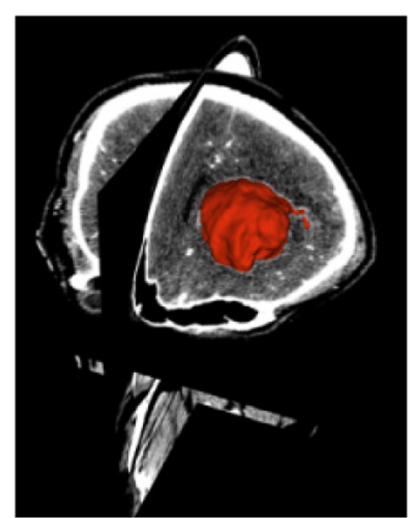

d

Fig. 1 Process of distinguishing the hematoma in the yellow circle. a Thresholding; $\mathbf{b}$ Using smoothing to optimize staining; $\mathbf{c}$ Using Islands to separate the hematoma; and $\mathbf{d}$ The fusion of the hematoma and the three-dimensional map

concept of the external center axis, we found the optimal external center axis suitable for this study. The maximum Euclidean distance in the hematoma point cloud is determined, and the line segment joining the two points is defined as the optimal external center axis.

\section{Algorithm of optimal external central axis extraction} The optimal external central axis can be obtained using a direct optimization algorithm [20]. However, a large amount of computation is required because the time complexity increases at the $\mathrm{N}^{2}$ level with an increasing quantity of point clouds. In the case of a largescale geometric model, the approach to obtain the central axis is excessively time consuming. Therefore, to reduce the computation redundancy, a point cloud clustering algorithm based on k-means is proposed to optimize the search space $[15,16]$. The algorithm presents strong adaptive ability, robustness, and low computation requirements. However, it does not consider the search method, and thus, it is not a suitable method for extracting the axis. Based on the above analysis, we propose the point cloud search arithmetic method for modified adaptive weighted particle swarm optimization [17-19, 26-30]. The algorithm reduces computational redundancy and greatly enhances the efficiency of searching axes in complex geometries. Our proposed algorithm is written in MATLAB (The MathWorks, Inc., MA).

\section{Algorithm flow}

The flow of the point cloud search arithmetic method for modified adaptive weighted particle swarm optimization based on k-means clustering is as follows:

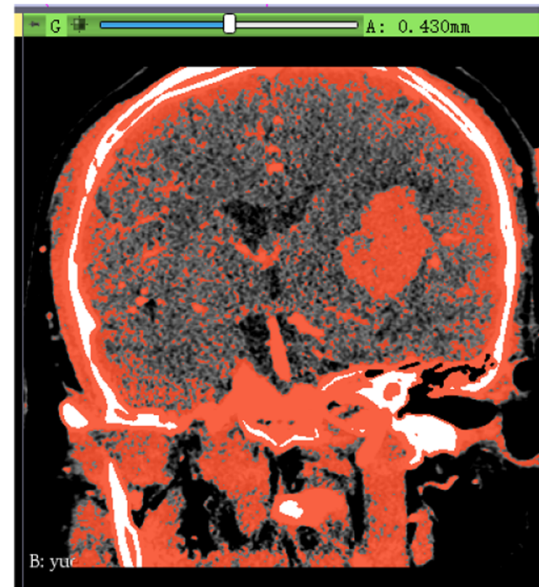

a

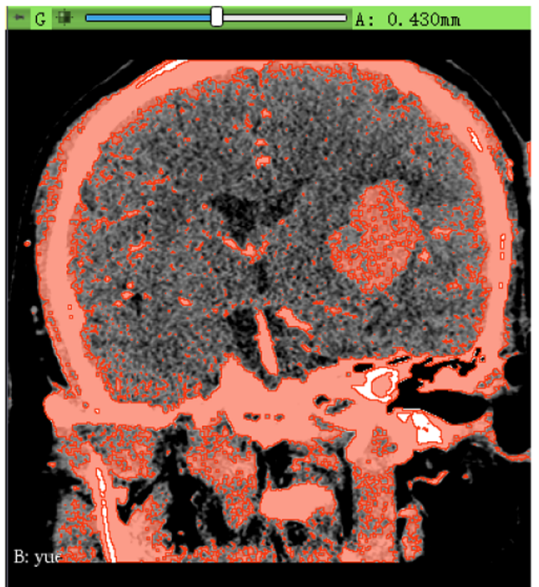

b

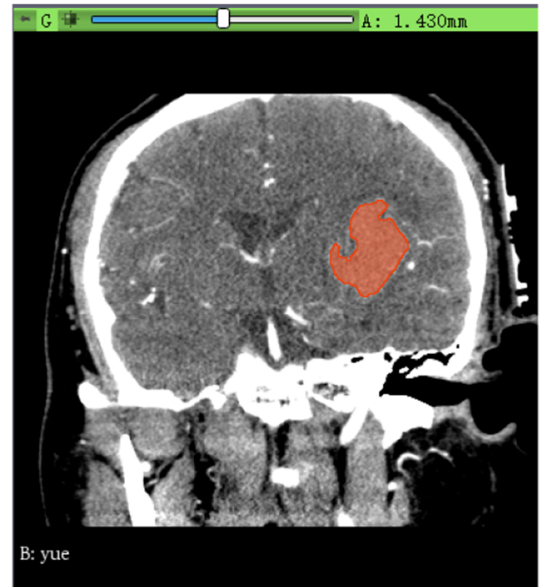

C

Fig. 2 Schematic diagram of hematoma extraction. a Mask effect; b Smoothing; and c hematoma segmentation 
Step 1. First, define the data set $D$ in $3 D$ Euclidean space and randomly select $n$ items $(n$ is the number of selected clusters) as centroids $C_{i}(i=1,2$, $\cdots, n)$, where each centroid is the clustering center of a category. Then, calculate the Euclidean distance between $C_{i}$ and $D_{j}(1,2, \cdots, k)$ in $D$. For example, if $D_{j}$ is the nearest to $C_{i}$, it is classified as cluster $i$.

Step 2. Through the first step, the data set $D$ is initially divided into $k$ classes. Calculate the mean value of each dimension of all data items in each cluster. A new centroid is formed and updated to the new centroid.

Step 3. Repeat steps 1 and 2 to obtain the new centroid until the centroid of each class no longer changes.

Step 4. Randomly initialize the particles in the solution space.

Step 5. Each particle updates its velocity and position by tracking its own and the group's optimal solution. The velocity updating formula of particle $i$ in the $d$ th dimension is as follows:

$$
v_{i d}^{k}=\omega v_{i d}^{k-1}+c_{1} r_{1}\left(\text { pbest }_{i d}-x_{i d}^{k-1}\right)+c_{2} r_{2}\left(\text { pbest }_{i d}-x_{i d}^{k-1}\right)
$$

The position updating formula is

$$
x_{i d}^{k}=x_{i d}^{k-1}+v_{i d}^{k-1}
$$

Repeat step 5 and incrementally iterate until the optimal position of the given threshold is found.

Note: $v_{i d}^{k}$ is the $d$-th dimensional component of the $k$ - th iterative particle $i$ flight velocity vector; $x_{i d}^{k}$ is the $d$-th dimensional component of the position vector; $c_{1} c_{2}$ is the maximum learning step parameter; $r_{1} r_{2}$ is the random function in the range of $[0-1]$; and $\omega$ is the inertia weight of the search, which has a considerable impact on the search scope and method.

Step 6. Compute the mean distance between $d_{1}, d_{2}$, $\cdots, \quad d_{n}: \quad D_{E X P}=\frac{2 * \sum D_{i j}}{k *(k-1)} \quad, \quad$ where $\quad D_{i j}=$ $\sqrt{\left(x_{i}-x_{j}\right)^{2}+\left(y_{i}-y_{j}\right)^{2}+\left(z_{i}-z_{j}\right)^{2}}$ denotes the distance between two clustering centers.

Step 7. Calculate and obtain the difference between $D_{i j}$ and $D_{E X P}: S_{i j}=D_{i j}-D_{E X P}$, where $\omega_{i j}$ denotes the ratio of $S_{i j}$ to $\sum S_{i j}$ as the generating weight of the small block $\omega_{i j}$ : $\omega_{i j}=\frac{S_{i j}}{\sum S_{i j}}$.

Step 8. Dynamically adjust the inertial weight of particles according to the NAIW method proposed by Li et al. [30].

$$
\omega_{i}^{j}=k \times \frac{\omega_{\max }-\omega_{\min }}{\max \left\{\Delta x_{i}^{t}\right\}}+\omega_{\min }
$$

$$
\begin{aligned}
& \Delta x_{i}=\sqrt{\sum_{d=1}^{D}\left(x_{i d}-G_{d}\right)^{2}} \\
& k=\frac{i t e r N u m-t}{\text { iterNum }}
\end{aligned}
$$

where $\omega_{\max }$ and $\omega_{\min }$ are 0.9 and 0.4 , respectively [20], $\Delta x_{i}$ is the distance between the ith particles and the extreme position of the group, and $k$ is the iteration coefficient. The NAIW method balances the weight of the particles under different circumstances, and it has advantages in convergence and robustness. Figure 3 is the flow diagram of the algorithm.

\section{Optimal path planning}

According to clinical demand and geometry symmetry, the straight line, where the optimal external axis is, is the path of the drainage tube into the brain. The multitube drainage tube can be divided into two subtubes and three subtube drainage tubes. The purpose of this study is to provide the corresponding optimal path planning for a specific multitube drainage tube [21]. The Table 1 is the explanation of symbols.

The known conditions can be determined by $M_{1}$ and $M_{2}$ According to the intersection coordinates $M_{1}\left(x_{1}, y_{1}, z_{1}\right)$ and $M_{2}\left(x_{2}, y_{2}, z_{2}\right)$, we obtain the parameter equation of the center axis as follows:

$$
\left\{\begin{aligned}
x & =x_{1}+\left(x_{2}-x_{1}\right) t \\
y & =y_{1}+\left(y_{2}-y_{1}\right) t, \text { where } 0 \leq t \leq 1(6) \\
z & =z_{1}+\left(z_{2}-z_{1}\right) t
\end{aligned}\right.
$$

The midpoint coordinates of $M_{1} M_{2}$ are $M_{1 / 2}=\left(\frac{x_{1}+x_{2}}{2}\right.$, $\left.\frac{y_{1}+y_{2}}{2}, \frac{z_{1}+z_{2}}{2}\right)$.

The distance from $M_{1}$ to $M_{2}$ is $d_{12}=$ $\sqrt{\left(x_{2}-x_{1}\right)^{2}+\left(y_{2}-y_{1}\right)^{2}+\left(z_{2}-z_{1}\right)^{2}}$.

The plane equation of $M_{3} M_{4}$ is

$$
\begin{aligned}
& \left(x_{2}-x_{1}\right)\left(x-\frac{x_{1}+x_{2}}{2}\right)+\left(y_{2}-y_{1}\right)\left(y-\frac{y_{1}+y_{2}}{2}\right) \\
& \quad+\left(z_{2}-z_{1}\right)\left(z-\frac{z_{1}+z_{2}}{2}\right) \\
& \quad=0
\end{aligned}
$$

The distance from $M_{3}$ to $M_{4}$ is $d_{34}=$ $\sqrt{\left(x_{4}-x_{3}\right)^{2}+\left(y_{4}-y_{3}\right)^{2}+\left(z_{4}-z_{3}\right)^{2}}$.

A comparison of the distance between $d_{12}$ and $d_{B_{i}}$ can offer directional evidence for puncture surgery. 


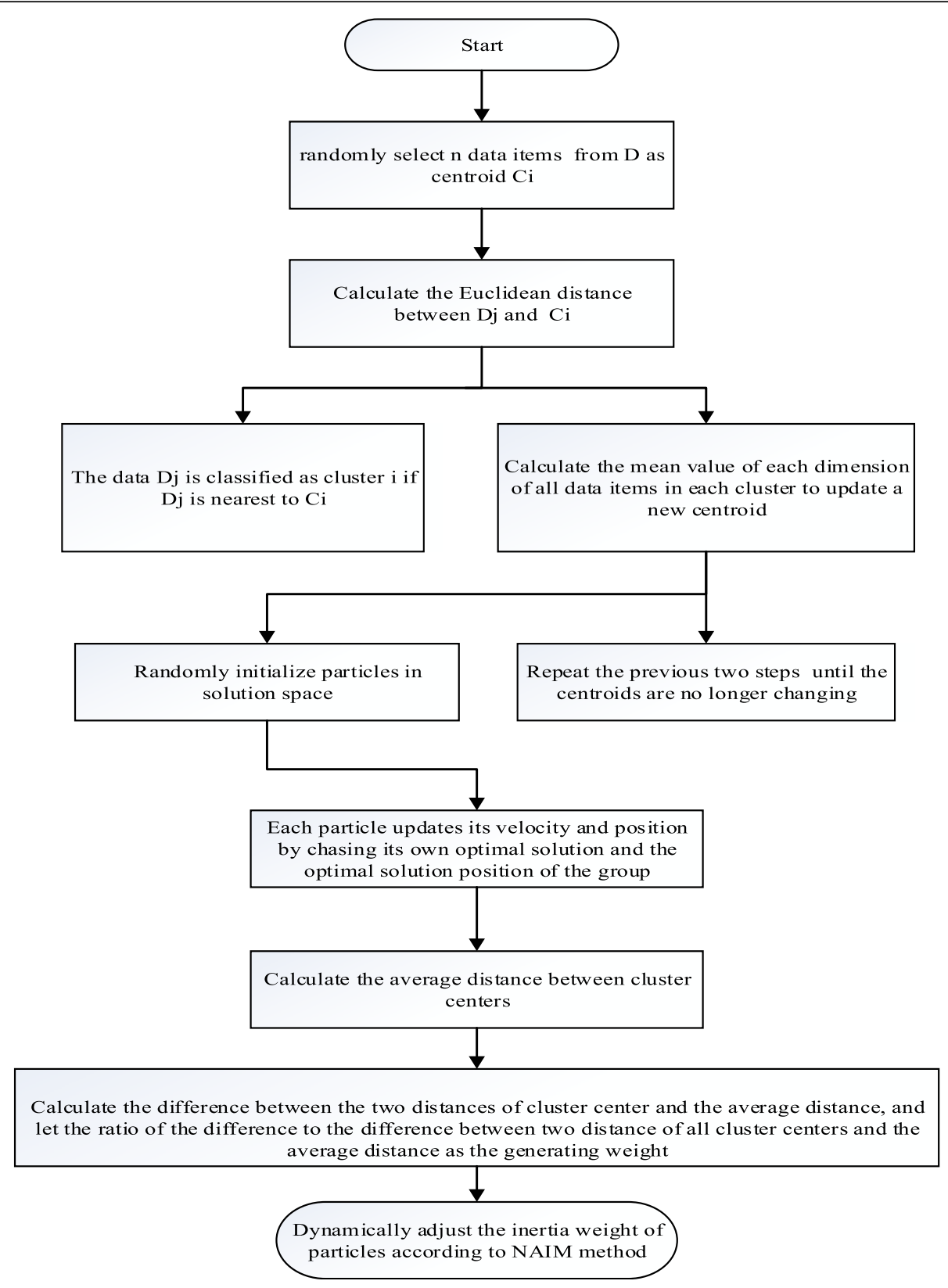

Fig. 3 Flow diagram of the algorithm to extract the optimal external middle axis

$$
\begin{cases}\frac{d_{B_{3}}-d_{B_{2}}}{2}+d_{B_{2}}<d_{12}, & \text { select three-subtubes } \\ \frac{d_{B_{3}}-d_{B_{2}}}{2}+d_{B_{2}} \geq d_{12}, & \text { select two-subtubes }\end{cases}
$$

The flow diagram of the bifurcation location algorithm of the drainage tube is as shown in Fig. 4.

\section{Optimal path planning of two subtube drainage tubes}

According to the clinical demand, the best position of the drainage tube in the hematoma is the midpoint between the two holes, coinciding with the midpoint of the central axis, that is, $M_{1 / 2}=\left(\frac{x_{1}+x_{2}}{2}, \frac{y_{1}+y_{2}}{2}, \frac{z_{1}+z_{2}}{2}\right)$, and the path of the main drainage tube coincides with the optimal external center axis.

\section{Calculating the coordinates of $B_{1}$ and $B_{2}$}

$$
t_{1}=\frac{\left|M_{1 / 2} B_{1}\right|+\left|M_{1 / 2} M_{1}\right|}{\left|M_{1} M_{2}\right|}=\frac{d_{12}+d_{O}-d-D}{2 d_{12}}
$$


Table 1 Notation

\begin{tabular}{ll}
\hline Symbol & Description \\
\hline$M_{1}\left(x_{1}, y_{1}, z_{1}\right), M_{2}\left(x_{2}, y_{2}\right.$, & The coordinate of the joint point of the optimal external axis and the boundary of the hematoma \\
$\left.z_{2}\right)$ & The distance from $M_{1}$ to $M_{2}$ \\
$d_{12}=\left|M_{1} M_{2}\right|$ & The maximum diameter of the vertical surface through $M_{1 / 2}$ in the hematoma \\
$d_{34}=\left|M_{3} M_{4}\right|$ & Ddenotes the diameter of the duct in the main tube; $d$ denotes the diameter of the hole in the subtube \\
$M_{3}\left(x_{3}, y_{3}, z_{3}\right), M_{4}\left(x_{4}, y_{4}\right.$, & The coordinates of the endpoints of $d_{34}$ in the hematoma \\
$\left.z_{4}\right)$ & The distance between the two central points of the holes \\
$D_{1} d$ & The distance between the two bifurcations \\
$d_{0}$ & The distance between the main probe tip and the $i-$ th bifurcation-tube probe tip (we define the first subtube to be nearest \\
$d_{B}$ & to the main probe tip)
\end{tabular}

Note: Only some common symbols are listed here; the rest are explained later

$$
t_{2}=\frac{\left|M_{1} B_{1}\right|+\left|B_{1} B_{2}\right|}{\left|M_{1} M_{2}\right|}=\frac{d_{12}+d_{O}+2 d_{B}-d-D}{2 d_{12}}
$$

Substituting $t_{1}, t_{2}$ into Eq. (6), we obtain the coordinates of $B_{1}\left(x_{B_{1}}, y_{B_{1}}, z_{B_{1}}\right)$ and $B_{2}\left(x_{B_{2}}, y_{B_{2}}, z_{B_{2}}\right)$.

\section{Calculating the direction vector of the subtube}

To find two line segments through $M_{3}$ with a fixed angle between this axis and the center axis, let the intersection points between the line segment and the axis be $M_{31}$, $M_{32}$; these points should meet the following limits:

$$
\begin{aligned}
& \frac{\overrightarrow{M_{31} M_{3}} \cdot \overrightarrow{M_{1} M_{2}}}{\left|\overrightarrow{M_{31} M_{3}}\right| *\left|\overrightarrow{M_{1} M_{2}}\right|}=-\frac{\sqrt{2}}{2} \\
& \left\{\begin{array}{c}
\frac{\overrightarrow{M_{32} M_{3}} \cdot \overrightarrow{M_{1} M_{2}}}{\left|\overrightarrow{M_{32} M_{3}}\right| *\left|\overrightarrow{M_{1} M_{2}}\right|}=\frac{\sqrt{2}}{2} \\
\overrightarrow{M_{31} M_{3}} \cdot \overrightarrow{M_{32} M_{3}}=0
\end{array}\right. \\
& t_{31}=\frac{d_{12}+2\left|M_{1 / 2} M_{3}\right|}{2 d_{12}} \\
& t_{32}=\frac{d_{12}-2\left|M_{1 / 2} M_{3}\right|}{2 d_{12}}
\end{aligned}
$$

Substituting $t_{31}, t_{32}$ into Eq. (6), we obtain the coordinates of $M_{31}, M_{32}$; the direction vector of subtubes are $\overrightarrow{M_{31} M_{3}}=\left(m_{31}, n_{31}, k_{31}\right)$ and $\overrightarrow{M_{3} M_{32}}=\left(m_{32}, n_{32}, k_{32}\right)$, and the parameter equation of the two subtubes is as follows:

\section{Scenario one:}

$$
\begin{aligned}
& x=x_{B_{1}}+m_{31} l \quad x=x_{B_{2}}+m_{32} l \\
& \left\{y=y_{B_{1}}+m_{31} l,\left\{y=y_{B_{2}}+m_{32} l\right.\right. \\
& z=z_{B_{1}}+m_{31} l \quad z=z_{B_{2}}+m_{32} l
\end{aligned}
$$

Scenario two:

$$
\begin{aligned}
x & =x_{B_{1}}+m_{32} l \quad x=x_{B_{2}}+m_{31} l \\
\{y & =y_{B_{1}}+m_{32} l,\left\{y=y_{B_{2}}+m_{31} l,(\text { where } l \geq 0)\right. \\
z & =z_{B_{1}}+m_{32} l \quad z=z_{B_{2}}+m_{31} l
\end{aligned}
$$

The intersection points between the four rays and the boundary of the hematoma are $B_{11}, B_{21}, B_{12}$, and $B_{22}$.
$\left\{\begin{array}{l}\left|B_{1} B_{11}\right|+\left|B_{2} B_{21}\right| \geq\left|B_{12} B_{1}\right|+\left|B_{22} B_{2}\right| \text { scenario one } \\ \left|B_{1} B_{11}\right|+\left|B_{2} B_{21}\right|<\left|B_{12} B_{1}\right|+\left|B_{22} B_{2}\right| \text { scenario two }\end{array}\right.$

In the first scenario, the coordinates of the probe tip of the subtube are as follows:

$$
B_{1}^{F}=\left(\frac{x_{B_{1}}+x_{B_{11}}}{2}, \frac{y_{B_{1}}+y_{B_{11}}}{2}, \frac{z_{B_{1}}+z_{B_{11}}}{2}\right), B_{2}^{F}=\left(\frac{x_{B_{2}}+x_{B_{21}}}{2}, \frac{y_{B_{2}}+y_{B_{21}}}{2},\right.
$$
$\left.\frac{z_{B_{2}}+z_{B_{21}}}{2}\right)(15)$

In the second scenario, the coordinates of the probe tip of the subtube are.

$$
\begin{aligned}
& B_{1}^{F}=\left(\frac{x_{B_{1}}+x_{B_{12}}}{2}, \frac{y_{B_{1}}+y_{B_{12}}}{2}, \frac{z_{B_{1}}+z_{B_{12}}}{2}\right), B_{2}^{F}=\left(\frac{x_{B_{2}}+x_{B_{22}}}{2}, \frac{y_{B_{2}}+y_{B_{22}}}{2},\right. \\
& \left.\frac{z_{B_{2}}+z_{B_{22}}}{2}\right)(16)
\end{aligned}
$$

The optimal path planning of three subtube drainage tubes According to the clinical demand, the best position of the drainage tube in the hematoma is the midpoint of the second hole, coinciding with the midpoint of the central axis, i.e., $M_{1 / 2}=\left(\frac{x_{1}+x_{2}}{2}, \frac{y_{1}+y_{2}}{2}, \frac{z_{1}+z_{2}}{2}\right)$, with the path of the main drainage tube coinciding with the optimal external center axis.

Calculating the coordinate of $B_{1}, B_{2}$, and $B_{3}$

$$
\begin{aligned}
& t_{11}=\frac{\left|M_{1} B_{1}\right|}{\left|M_{1} M_{2}\right|}=\frac{d_{12}-d-D}{2 d_{12}} \\
& t_{12}=\frac{\left|M_{1} B_{2}\right|}{\left|M_{1} M_{2}\right|}=\frac{d_{12}+2 d_{O}-d-D}{2 d_{12}} \\
& t_{13}=\frac{\left|M_{1} B_{3}\right|}{\left|M_{1} M_{2}\right|}=\frac{d_{12}+4 d_{O}-d-D}{2 d_{12}}
\end{aligned}
$$

Substituting $t_{11}, t_{12}$ and $t_{13}$ into Eq. (6), we obtain the coordinates $B_{1}\left(x_{B_{1}}, y_{B_{1}}, z_{B_{1}}\right), B_{2}\left(x_{B_{2}}, y_{B_{2}}, z_{B_{2}}\right)$, and $B_{3}\left(x_{B_{3}}\right.$, $\left.y_{B_{3}}, z_{B_{3}}\right)$. 


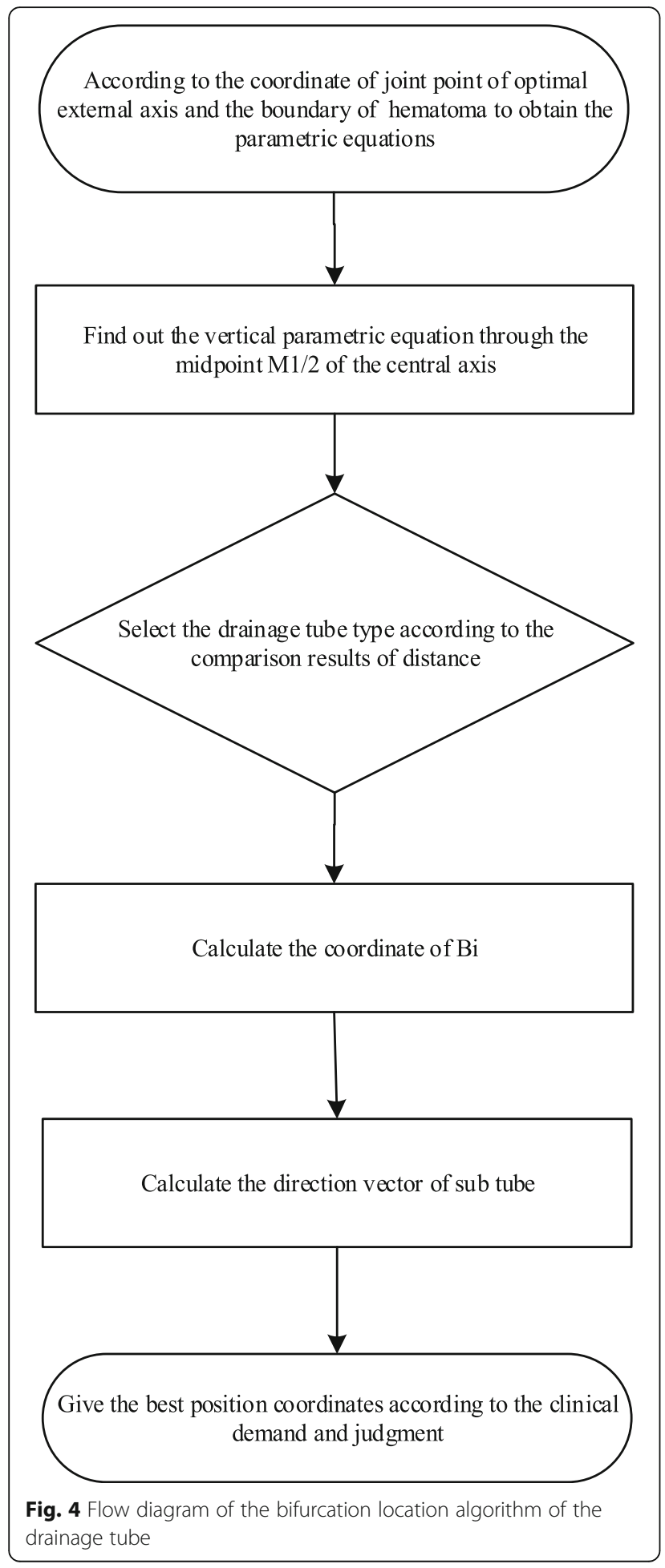

\section{Calculating the direction vector of the subtube}

To find a line segment through $M_{3}$ with a fixed angle between the axis $M_{1} M_{2}$ and the center axis, let the intersection points between the line segment and the axis be $M_{31}\left(x_{31}, y_{31}, z_{31}\right)$; they should satisfy the following limits:

$$
\left\{\begin{array}{l}
\frac{\overrightarrow{M_{31} M_{3}} \cdot \overrightarrow{M_{2} M_{1}}}{\left|\overrightarrow{M_{31} M_{3}}\right| *\left|\overrightarrow{M_{2} M_{1}}\right|}=\frac{\sqrt{2}}{2}\left|\overrightarrow{M_{31} M_{3}}\right|=\mid \overrightarrow{M_{3} M_{1 / 2}} \\
\mid x_{31}=x_{1}+\frac{\left|\overrightarrow{M_{3} M_{1 / 2}}\right|+\left|\overrightarrow{M_{1} M_{1 / 2}}\right|}{\left|\overrightarrow{M_{1} M_{2}}\right|}\left(x_{2}-x_{1}\right) \\
y_{31}=y_{1}+\frac{\left|\overrightarrow{M_{3} M_{1 / 2}}\right|+\left|\overrightarrow{M_{1} M_{1 / 2}}\right|}{\left|\overrightarrow{M_{1} M_{2}}\right|}\left(y_{2}-y_{1}\right) \\
z_{31}=z_{1}+\frac{\left|\overrightarrow{M_{3} M_{1 / 2}}\right|+\left|\overrightarrow{M_{1} M_{1 / 2}}\right|}{\left|\overrightarrow{M_{1} M_{2}}\right|}\left(z_{2}-z_{1}\right)
\end{array}\right.
$$

We obtain $\overrightarrow{M_{31} M_{3}}=\left(x_{3}-x_{31}, y_{3}-y_{31}, z_{3}-z_{31}\right)=\left(m_{1}\right.$, $\left.n_{1}, k_{1}\right)$.

Because the angle between two subtubes and the angle between the subtube and the main tube are fixed values, we can calculate the other two direction vectors of the subtubes based on the geometrical relationship.

$$
\begin{aligned}
\overrightarrow{B_{1}^{2}}= & \overrightarrow{M_{31} M_{1 / 2}}+\frac{1}{2} \overrightarrow{M_{3} M_{4}} \\
& +\frac{\sqrt{3}}{2}\left(\overrightarrow{M_{3} M_{31}} \times \overrightarrow{M_{31} M_{1 / 2}}\right) \\
= & \left(m_{2}, n_{2}, k_{2}\right) \\
\overrightarrow{B_{1}^{3}}= & \overrightarrow{M_{31} M_{1 / 2}}+\frac{1}{2} \overrightarrow{M_{3} M_{4}} \\
& +\frac{\sqrt{3}}{2}\left(\overrightarrow{M_{1 / 2} M_{31}} \times \overrightarrow{M_{31} M_{3}}\right) \\
= & \left(m_{3}, n_{3}, k_{3}\right)
\end{aligned}
$$

The parametric equation of the three subtubes is as follows.

$$
\begin{aligned}
& x=x_{B_{i}}+m_{j} t \\
& \left\{y=y_{B_{i}}+n_{j} t \text {, where } i=1,2,3 ; j=1,2,3\right. \\
& z=z_{B_{i}}+k_{j} t
\end{aligned}
$$

There are three cases for the direction vector of each subtube; let the coordinates of the subtube and the boundary of the hematoma be $B_{11}, B_{12}, B_{13} ; B_{21}, B_{22}$, $B_{23} ; B_{31}, B_{32}, B_{33}$.

$$
d_{B_{i}}=\max \left(\left|B_{1} B_{1 i}\right|+\left|B_{2} B_{2 j}\right|+\left|B_{3} B_{3 k}\right|\right),\left\{\begin{array}{c}
i, j, k=1,2,3 \\
i \neq j \neq k
\end{array}\right.
$$

When we find the maximum value $d_{B_{i}}$, the coordinates of the bifurcation subtube probe in hematoma can be obtained as $\frac{B_{1} B_{1 i}}{2}, \frac{B_{2} B_{2 i}}{2}$, and $\frac{B_{3} B_{3 k}}{2}$. 

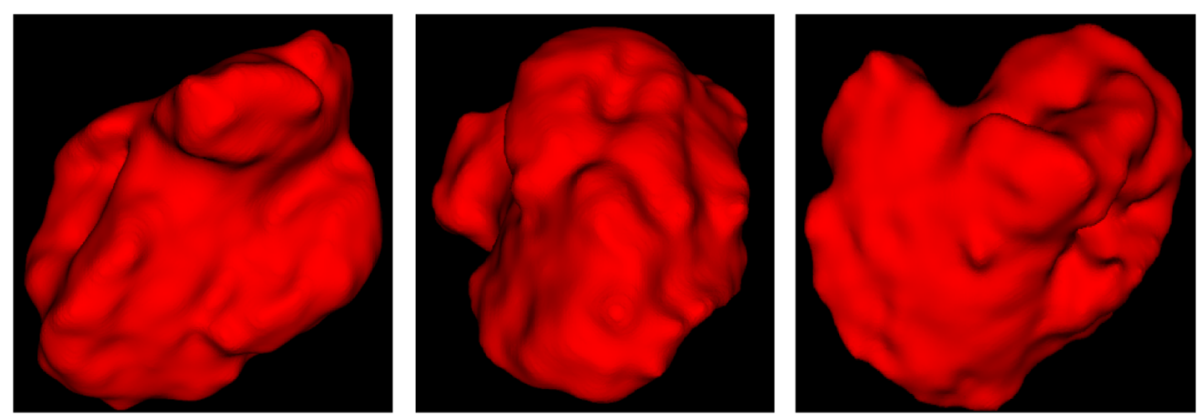

Fig. 5 Three-dimensional intracranial hematoma model

\section{Results and discussion}

According to the CT data, the hematoma area was segmented, and an independent three-dimensional diagram was obtained, as shown in Fig. 5.

The STL format of a three-dimensional point cloud is obtained by hematoma modeling. Figure 6 shows the three-dimensional point cloud diagram in the hematoma area, and Fig. 7 shows the triangulation diagram of this area [24].

In this case, the point cloud search arithmetic method for modified adaptive weighted particle swarm optimization to search the hematoma region uses 81,226 point-chains. The coordinates of the intersection between the central axis and the boundary of the hematoma are $M_{1}\left(x_{1}, y_{1}, z_{1}\right)$ and $M_{2}\left(x_{2}, y_{2}\right.$, $\left.z_{2}\right)$. The optimal external central axis of the hematoma is shown in Fig. 8. The intersection points of the central axis and the boundary of the hematoma are $M_{1}(28.555,9.623,7.123)$ and $M_{2}(43.384,21.295,48.887)$.

Therefore, the midpoint coordinates of the center axis $M_{1} M_{2}$ are $M_{1 / 2}(35.970,5.836,28.010)$, and the distance from $M_{1}$ to $M_{2}$ is $d_{12}=\left|M_{1} M_{2}\right| \approx 54.0377$. Figure 8 is optimal external central axis of the hematoma.
The route planning for the puncture operation is as follows:

Because $\frac{d_{B_{3}}-d_{B_{2}}}{2}+d_{B_{2}}=35<d_{12} \approx 54.0377$, it is suggested that doctors choose three subtube drainage tubes when performing puncture operations. From the coordinate of the center axis, the plane equation through $M_{3} M_{4}$ can be obtained as

$$
\begin{gathered}
14.82919(x-35.969525)+30.91862 \\
(y-5.83595)+71.763726 \\
(z-28.009754)=0 .
\end{gathered}
$$

According to the point cloud search arithmetic method for modified adaptive weighted particle swarm optimization, we obtain the coordinates as $M_{3}(26.201 .451,18.457) \quad$ and $M_{4}(36.353,-7.797$, 37.816), and the distance from $M_{3}$ to $M_{4}$ is $d_{34}=$ 38.134751 .

Through the above algorithm process, the coordinates of the three bifurcation points can be calculated

$$
\begin{aligned}
& B_{1}(35.18971256,4.219077228,25.81685749), \\
& B_{2}(37.93409287,9.939157835,33.54479557),
\end{aligned}
$$
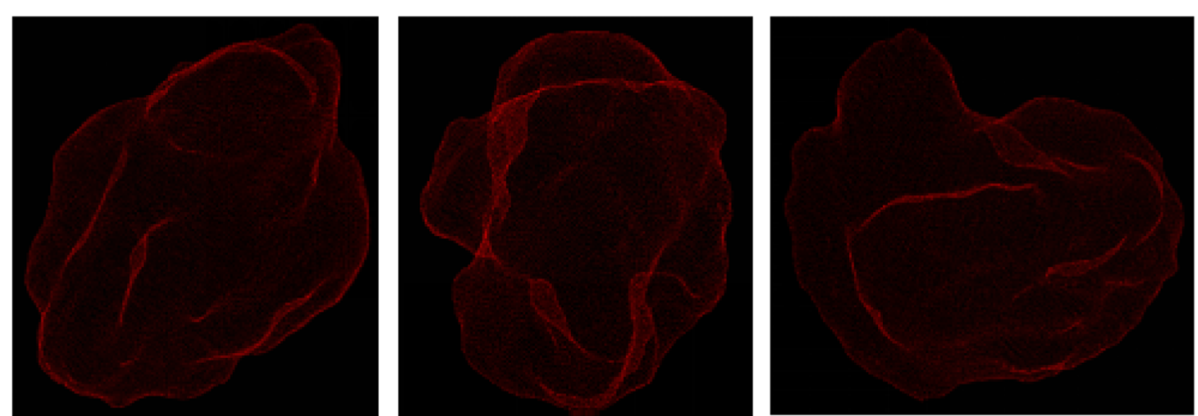

Fig. 6 The point cloud model 

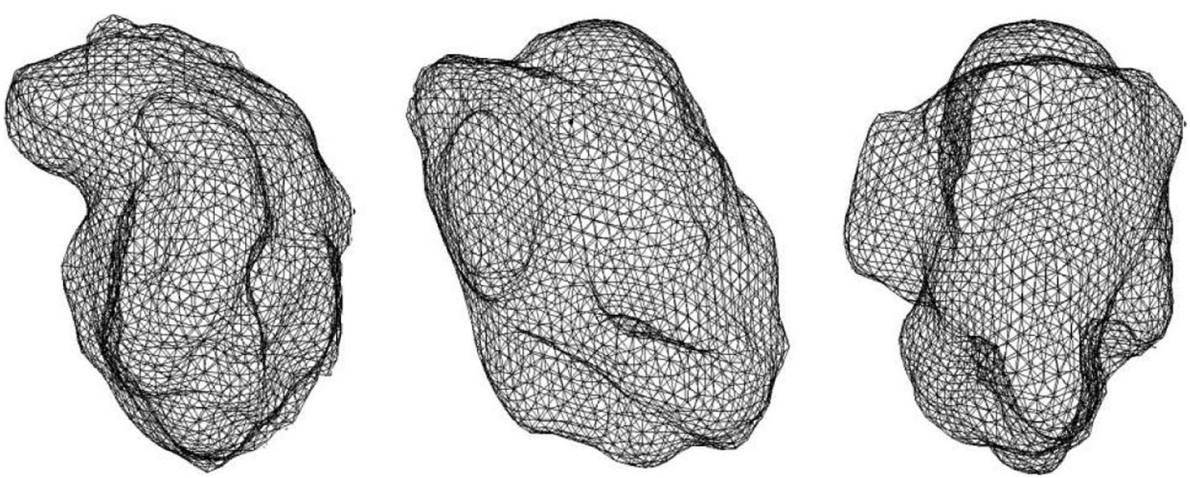

Fig. 7 Schematic diagram of triangulation of the hematoma in STL format

$$
B_{3}(40.67847319,15.65923845,41.27273366) .
$$

The three direction vectors of the subtube are as follows:

$$
\begin{aligned}
\overrightarrow{M_{31} M_{3}} & =(-0.296512566,-24.3104924,-4.838389244), \\
\overrightarrow{B_{1}^{2}} & =(3.125640267,-0.881458887,-10.79916805), \\
\overrightarrow{B_{1}^{3}} & =(-6.200200368,-1.216682803,-3.497583415) .
\end{aligned}
$$

There are three cases for the direction vector of each subtube; let the coordinates of the subtube and the boundary of the hematoma be $B_{1,1}, B_{1,2}, B_{1,3} ; B_{2,1}, B_{2}$, ${ }_{2}, B_{2,3} ; B_{3,1}, B_{3,2}, B_{3,3}$. The maximum value from the bifurcation point to the boundary of the hematoma is

$$
d_{B_{i i}}=\max \left(\left|B_{1} B_{1, i}\right|+\left|B_{2} B_{2, j}\right|+\left|B_{3} B_{3, k}\right|\right),\left\{\begin{array}{c}
i, j, k=1,2,3 \\
i \neq j \neq k
\end{array} .\right.
$$

The coordinates of the bifurcation probe to reach the hematoma area are

$$
\begin{aligned}
& B_{11}=(35.11854955,-1.615440948,24.6556440), \\
& B_{22}=(39.80947703,9.410282503,27.0652947), \\
& B_{33}=(38.19839304,15.17256533,39.8737003) .
\end{aligned}
$$

Figure 9 is a schematic diagram of the path planning process.

In this study, 3D Slicer software was used to reconstruct a three-dimensional model of an intracranial hematoma, and an improved algorithm based on clustering and adaptive particle swarm optimization to extract the optimal external central axis of the hematoma was proposed. According to the characteristics of the drainage tube and the specific morphology of the intracranial hematoma, optimal path planning under ideal conditions was performed, and the preoperative simulation was provided, which will be useful in clinical medicine.

The bifurcation localization algorithm of the drainage tube proposed in this study assumes an ideal state. It is necessary to consider the distribution of peripheral

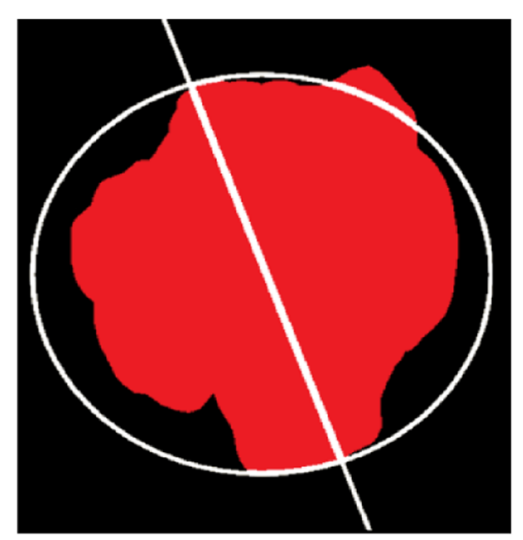

a

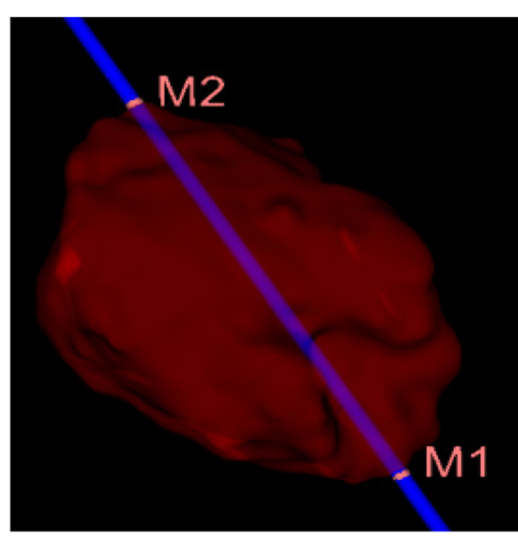

b

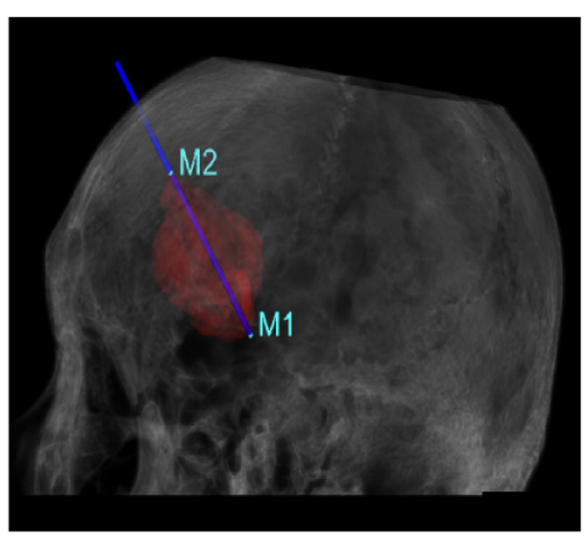

$\mathrm{c}$

Fig. 8 Optimal external central axis of the hematoma. a Two-dimensional projection; b Three-dimensional projection; and $\mathbf{c}$ The exact position in skull 


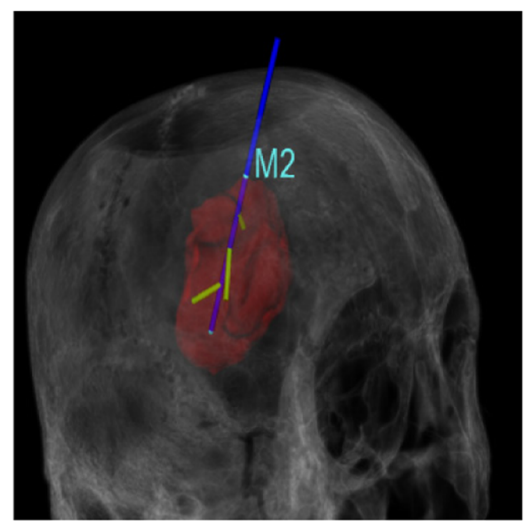

(a)

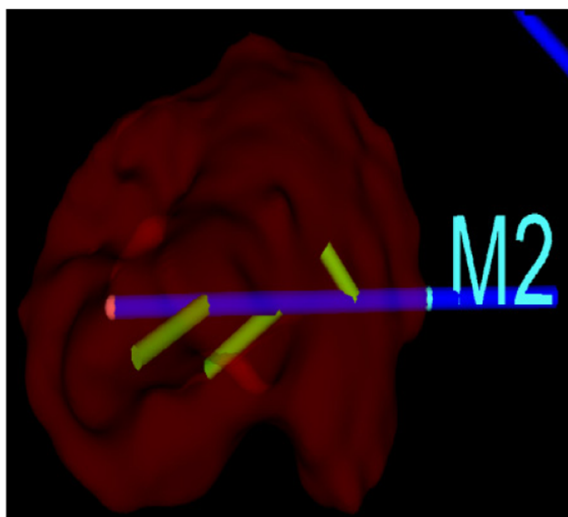

(b)

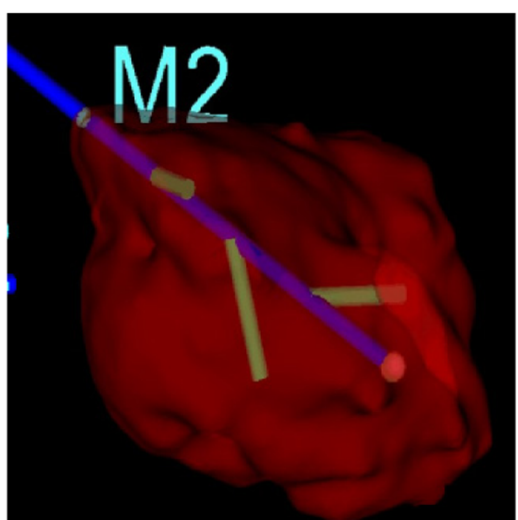

(c)

Fig. 9 Route planning. $M_{2}$ denotes the accurate position of the puncture point

nerves and blood vessels and the specific location of the hematoma in the brain to apply this method to clinical medicine. The focus of subsequent research work will be to optimize the algorithm by considering the above factors, to implement the diffusion model and simulation of the drug injection, to plan the optimal surgical project, and to provide a dynamic simulation before operation.

\section{Abbreviations}

$\mathrm{CT}$ : Computerized tomography; ROI: Region of interest

\section{Acknowledgments}

Authors thank Tangshan Gongren Hospital for their support of original data.

\section{Authors' contributions}

All authors read and approved the final manuscript. Conceptualization, JChang, JCui, QP; methodology, QP and WZ; software, WZ and XZ; formal analysis, QP; resources, JChang; data curation, JChang and JCui; writing —original draft preparation, QP; writing_-review and editing, QP; visualization, QP and WZ; supervision, JChang and JCui; funding acquisition, JChang.

\section{Funding}

This research was funded by the National Science Foundation of China, Nos. 51674121 and 61702184; the Returned Overseas Scholar Funding of Hebei Province, No. C2015005014; the Hebei Key Laboratory of Science and Application, and Tangshan Innovation Team Project, No. 18130209B.

\section{Availability of data and materials}

The datasets used and/or analysed during the current study are not publicly available due to personal privacy but are available from the corresponding author on reasonable request.

Supplementary Materials: The following are available online at www.mdpi. $\mathrm{com} / \mathrm{xxx} / \mathrm{s} 1$, Figures, Tables

\section{Competing interests}

The authors declare that they have no competing interests.

Received: 3 September 2019 Accepted: 4 December 2019

Published online: 14 January 2020

\section{References}

1. Fiorella D, Zuckerman SL, Khan IS, KumarN G, Mocco J (2015) Intracerebral hemorrhage: a common and devastating disease in need of better treatment. World Neurosurg 84(4):1136-1141. https://doi.org/10.1016/j. wneu.2015.05.063

2. Qureshi Al, Tuhrim S, Broderick JP, Batjer H, Hondo H, Hanley DF (2001) Spontaneous intracerebral hemorrhage. N Engl J Med 344(19):1450-1460. https://doi.org/10.1056/NEJM200105103441907

3. Xiao FR, Chiang IJ, Wong JM, Tsai YH, Huang KC, Liao CC (2011) Automatic measurement of midline shift on deformed brains using multiresolution binary level set method and Hough transform. Comput Biol Med 41(9):756762. https://doi.org/10.1016/j.compbiomed.2011.06.011

4. Samadani U, Rohde V (2009) A review of stereotaxy and lysis for intracranial hemorrhage. Neurosurg Rev 32(1):15-22. https://doi.org/10.1007/s10143-008-0175-Z

5. Wang GQ, Li SQ, Huang YH, Zhang WW, Ruan WW, Qin JZ et al (2014) Can minimally invasive puncture and drainage for hypertensive spontaneous basal ganglia intracerebral hemorrhage improve patient outcome: a prospective non-randomized comparative study. Mil Med Res 1:10. https:// doi.org/10.1186/2054-9369-1-10

6. Zhou HG, Zhang Y, Liu L, Han X, Tao YH, Tang YP et al (2011) A prospective controlled study: minimally invasive stereotactic puncture therapy versus conventional craniotomy in the treatment of acute intracerebral hemorrhage. BMC Neurol 11:76. https://doi.org/10.1186/1471-2377-11-76

7. Delcourt C, Anderson C (2012) Acute intracerebral haemorrhage: grounds for optimism in management. J Clin Neurosci 19(12):1622-1626

8. Backlund EO, von Holst H (1978) Controlled subtotal evacuation of intracerebral haematomas by stereotactic technique. Surg Neurol 9(2):99101. https://doi.org/10.1016/j.jocn.2012.05.018

9. Yan YF, Ru DW, Du JR, Shen X, Wang ES, Yao HB (2015) The clinical efficacy of neuronavigation-assisted minimally invasive operation on hypertensive basal ganglia hemorrhage. Eur Rev Med Pharmacol Sci 19(14):2614-2620

10. Cao YF (2019) Introduction of 3D slicer. https://www.slicercn.com/?page_ id=485. Accessed 10 Apr 2019

11. Pinter C, Lasso A, Pieper S, Plesniak W, Kikinis R, Miller J (2019) Segment editor. https://slicer.readthedocs.io/en/latest/user_guide/module_ segmenteditor.html. Accessed 10 Apr 2019

12. Zhang XL, Zhang KX, Pan QL, Chang JC (2019) Three-dimensional reconstruction of medical images based on 3D slicer. J Complexity Health Sci 2(1):1-12. https://doi.org/10.21595/chs.2019.20724

13. Pszczolkowski S, Law ZK, Gallagher RG, Meng DW, Swienton DJ, Morgan PS et al (2019) Automated segmentation of haematoma and perihaematomal oedema in MRI of acute spontaneous intracerebral haemorrhage. Comput Biol Med 106:126-139. https://doi.org/10.1016/j.compbiomed 2019.01.022

14. Zhang J, Yan CH, Chui CK, Ong SH (2010) Fast segmentation of bone in CT images using 3D adaptive thresholding. Comput Biol Med 40(2):231-236. https://doi.org/10.1016/j.compbiomed.2009.11.020

15. Wagstaff K, Cardie C, Rogers S, Schrodl S (2001) Constrained k-means clustering with background knowledge. In: Abstracts of the 18th international conference on machine learning. Morgan Kanufman Press, San Francisco

16. Likas A, Vlassis N, Verbeek JJ (2003) The global k-means clustering algorithm. Pattern Recogn 36(2):451-461. https://doi.org/10.1016/S00313203(02)00060-2 
17. Bai QH (2010) Analysis of particle swarm optimization algorithm. Computer Inf Sci 3(1):180-184. https://doi.org/10.5539/cis.v3n1p180

18. Xing H, Pan XJ (2018) Application of improved particle swarm optimization in system identification. In: Abstracts of 2018 Chinese control and decision conference. IEEE, Shenyang. https://doi.org/10.1109/CCDC.2018.8407336

19. Su Q, Yang $L H$, Fu YG, Wu YJ, Gong XT (2014) Parameter training approach based on variable particle swarm optimization for belief rule base. J Comput Appl 34(8):2161-2165

20. Khong SZ, Nešić D, Manzie C, Tan Y (2013) Multidimensional global extremum seeking via the DIRECT optimisation algorithm. Automatica 49(7): 1970-1978. https://doi.org/10.1016/j.automatica.2013.04.006

21. Cui JZ, Cui Y (2019) The multifunctional drainage tube with multi-tube for intracranial hematoma. CN patent CN208405725U

22. Siddiqi K, Pizer SM (2008) Medial representations: mathematics, algorithms and applications. Springer, Dordrecht. https://doi.org/10.1007/978-1-4020-8658-8

23. Wang Y, Li J, Chen S (2011) A novel method of extracting 3D blood vessel images axis based on energy constraint equation. J Comput Inf Syst 7(4): 1319-1327

24. Zhong YJ, Chen FL (2018) Computing medial axis transformations of $2 D$ point clouds. Graph Model 97:50-63. https://doi.org/10.1016/j.gmod.2018.03.004

25. Zhong YJ (2018) Computing medial axis transformations of the geometric model. J Comput Aided Des Comput Graph 30(8):1394-1412. https://doi. org/10.3724/SP.J.1089.2018.16790

26. Feng CS, Cong S, Feng XY (2007) A new adaptive inertia weight strategy in particle swarm optimization. In: Abstracts of 2007 IEEE congress on evolutionary computation. IEEE, Singapore, pp 25-28. https://doi.org/10. 1109/CEC.2007.4425017

27. Zhang LM, Tang YG, Hua CC, Guan XP (2015) A new particle swarm optimization algorithm with adaptive inertia weight based on Bayesian techniques. Appl Soft Comput 28:138-149. https://doi.org/10.1016/j.asoc. 2014.11.018

28. Li ZQ, Zheng H, Pei CM (2010) Particle swarm optimization algorithm based on adaptive inertia weight. In: Abstracts of the 2010 2nd international conference on signal processing systems. IEEE, Dalian, pp 5-7

29. Ao YC, Shi YB, Zhang W, Li YJ (2014) Improved particle swarm optimization with adaptive inertia weight. J Univ Electron Sci Technol China 43(6):874-880

30. Li LS, Zhang XJ (2018) New chaos particle swarm optimization based on adaptive inertia weight. Comput Eng Appl 54(9):139-144

\section{Publisher's Note}

Springer Nature remains neutral with regard to jurisdictional claims in published maps and institutional affiliations.

\section{Submit your manuscript to a SpringerOpen ${ }^{\circ}$ journal and benefit from:}

- Convenient online submission

- Rigorous peer review

- Open access: articles freely available online

- High visibility within the field

- Retaining the copyright to your article

Submit your next manuscript at $\boldsymbol{\nabla}$ springeropen.com 